Pacific Journal of Mathematics

EXTENSION FUNCTIONS FOR RANK 2, TORSION FREE 


\title{
EXTENSION FUNCTIONS FOR RANK 2, TORSION FREE ABELIAN GROUPS
}

\author{
ERIC M. Friedlander
}

The set of isomorphism classes of rank 2, torsion free abelian groups with a pure subgroup isomorphic to a given rank 1 group is shown to be in natural 1-1 correspondence with the set of pairs consisting of a quotient type and a type of an extension function. In terms of these invariants, necessary and sufficient conditions are determined for such a group to be homogeneous or to admit a pure cyclic subgroup. Moreover, this 1-1 correspondence has an explicit inverse, so that examples are readily obtained.

Our method is to combine the Korosh-Malcev-Derry matrix classification (see [2] for this and other well known aspects of the theory of abelian groups which we employ) with some elementary observations converning abelian extensions of rank 1, torsion free abelian groups. We begin $\S 1$ by identifying $\operatorname{Ext}_{\mathrm{z}}(X, Y)$ for rank 1, torsion free abelian groups $X$ and $Y$ in terms of "extension functions." A KoroshMalcev-Derry matrix sequence for the total group of such an extension is readily given in terms of an extension function. Moreover, an extension function explicitly determines a subgroup of $Q \oplus Q$. We obtain an explicit necessary condition for two extension functions to determine isomorphic total groups, as well as express the KoroshMalcev-Derry matrix conditions in terms of extension functions.

In $\$ 2$, we explicate the $1-1$ correspondence asserted above. We then "list" all homogeneous, rank 2, torsion free abelian groups of a given type. We also determine in terms of our invariants whether or not a rank 2, torsion free abelian group admits a pure cyclic subgroup.

We gratefully acknowledge many helpful conversations with $\mathrm{C}$. Miller. Moreover, the referee's suggestion of generalizing an eariler version of this work proved most valuable. We refer the interested reader to ([1]) for a detailed study of rank 2, torsion free abelian groups up to quasi-isomorphism and to ([2]) for a survey of the literature on torsion free abelian groups.

1. Extension functions. A sequence $\left(a_{1}, \cdots, a_{n}, \cdots\right)$ with each $a_{i}$ an extended nonnegative integer, $0 \leqq a_{i} \leqq \infty$, is called a characteristic. Two characteristics, $\left(a_{1}, \cdots, a_{n}, \cdots\right)$ and $\left(b_{1}, \cdots, b_{n}, \cdots\right)$, are said to have the same type if and only if $\Sigma_{i}\left(a_{i}-\right.$ $\left.b_{i}\right)^{2}<\infty$. If $A$ is a torsion free abelian group, then the characteristic of any nonzero element $x$ in $A, \operatorname{char}(x)=\left(a_{1}, \cdots, a_{n}, \cdots\right)$, is defined by 
$a_{i}=\operatorname{char}_{i}(x)=\sup \left\{k: p_{i}^{-k} x\right.$ in $\left.A\right\}$, where $p_{i}$ denotes the $i$ th prime. Two torsion free, rank 1 abelian groups, $X$ and $Y$, are isomorphic if and only if the type of $\operatorname{char}(x)$ equals the type of char $(y)$ for any nonzero elements $x$ and $y$ of $X$ and $Y$ respectively.

We first explicitly compute $\operatorname{Ext}_{\mathbf{z}}^{1}(X, Y)$ for any pair of rank 1, torsion free abelian groups $X$ and $Y$.

Proposition 1.1. Let $X$ and $Y$ be rank 1, torsion free abelian groups and let $x$ in $X$ and $y$ in $Y$ be nonzero elements. Let $\left(a_{1}, \cdots, a_{n}, \cdots\right)=\operatorname{char}(x)$ and $\left(b_{1}, \cdots, b_{n}, \cdots\right)=\operatorname{char}(y)$. Then there ex ists an isomorphism

$$
\varphi(X, x ; Y, y): \operatorname{Ext}_{\mathbf{z}}(X, Y) \rightarrow \operatorname{coker}\left(\theta_{y}: Y \rightarrow \Pi^{\prime} Z / p_{i}^{a_{i}}\right)
$$

where $\Pi^{\prime}$ denotes the product over all $i$ with $b_{i}<\infty$, where $\mathbf{Z} / p_{i}^{a_{i}}$ denotes the $p_{i}$-adic integers $\mathbf{Z}_{p_{i}}$ if $a_{i}=\infty$, and where $\theta_{y}(m / n \cdot y)=$ $\left(m / n \cdot p_{1}^{b_{1}}, \cdots, m / n \cdot p_{n}^{b_{n}}, \cdots\right)$. Moreover, $\varphi(X, x ; Y, y)$ is natural for maps $\left(X^{\prime}, x^{\prime}\right) \rightarrow(X, x)$ and maps $\left(Y^{\prime}, y^{\prime}\right) \rightarrow(Y, y)$.

Proof. Let $Y \rightarrow Q$ be defined by sending $y$ to 1 . Since $Q$ and $Q / Y$ are divisible abelian groups, $\operatorname{Ext}_{\mathrm{z}}(X, Y)$ is naturally isomorphic to $\operatorname{coker}(\operatorname{Hom}(X, Q) \rightarrow \operatorname{Hom}(X, Q / Y))$. Applying the serpent lemma to the following map of short exact sequences

$$
\begin{aligned}
& 0 \rightarrow \operatorname{Hom}(X / \mathbf{Z} \cdot x, Q) \rightarrow \operatorname{Hom}(X, Q) \rightarrow \operatorname{Hom}(\mathbf{Z} \cdot x, Q) \rightarrow 0 \\
& 0 \rightarrow \operatorname{Hom}(X / Z \cdot x, Q / Y) \rightarrow \operatorname{Hom}(X, Q / Y) \rightarrow \operatorname{Hom}(\mathbf{Z} \cdot x, Q / Y) \rightarrow 0
\end{aligned}
$$

we conclude that $\operatorname{Ext}_{\mathbf{z}}(X, Y) \stackrel{\sim}{\rightarrow} \operatorname{coker}(Y \rightarrow \operatorname{Hom}(X / Z \cdot x, Q / Y))$.

Let $\mu(p, a)$ denote the cyclic subgroup of $Q / Z$ generated by $1 / p^{a}$ (let $\left.\mu(p, \infty)=\lim _{n} \mu(p, n)\right)$. Then

$$
X / \mathbf{Z} \cdot x \stackrel{\sim}{\rightarrow} \oplus \mu\left(p_{i}, a_{i}\right) \quad \text { and } \quad Q / Y \stackrel{\sim}{\rightarrow} \bigoplus^{\prime} \mu\left(p_{i}, \infty\right)
$$

where the latter sum is taken over all $i$ with $b_{i}<\infty$. Thus

$$
\operatorname{Hom}(X / \mathbf{Z} \cdot x, Q / Y) \stackrel{\sim}{\rightarrow} \operatorname{Hom}\left(\bigoplus \mu\left(p_{i}, a_{i}\right), \oplus^{\prime} \mu\left(p_{i}, \infty\right)\right) \stackrel{\sim}{\rightarrow} \Pi^{\prime} \mathbf{Z} / p_{i^{\prime}}^{a_{i}}
$$

The map $Y \rightarrow \operatorname{Hom}(X / Z \cdot x, Q / Y)$ is easily checked to send $y$ to the sequence $\left\{p_{i}^{b_{i}}\right\}$ under these identifications.

To check the naturality of $\varphi(X, x ; Y, y)$ for a map $f:\left(X^{\prime}, x^{\prime}\right) \rightarrow(X, x)$, one must verify the commutativity of the following square: 


$$
\begin{gathered}
\operatorname{Ext}_{\mathbf{Z}}(X, Y) \stackrel{\varphi(X, x ; Y, y)}{\longrightarrow} \operatorname{coker}\left(\theta_{y}: Y \rightarrow \Pi^{\prime} \mathbf{Z} / p_{i}^{a_{i}}\right) \\
\downarrow \operatorname{Ext}_{\mathrm{z}}(f, \mathbf{Z}) \quad \downarrow \pi \\
\operatorname{Ext}_{\mathbf{z}}\left(X^{\prime}, Y\right) \stackrel{\varphi\left(Z^{\prime}, x^{\prime} ; Y, y\right)}{\longrightarrow} \operatorname{coker}\left(\theta_{y}: Y \rightarrow \Pi^{\prime} Z / p_{i}^{a_{i}}\right)
\end{gathered}
$$

where $\pi$ is induced by the factor by factor projection map. This verification, and the corresponding verification for naturality with respect to maps $g:\left(Y^{\prime}, y^{\prime}\right) \rightarrow(Y, y)$, are routine.

For a given $(Y, y)$, we call $f$ in $\Pi^{\prime} Z / p_{i}^{a_{i}}$ an "extension function" for the corresponding extension $\varphi(X, x ; Y, y)^{-1}(\bar{f})$ in $\operatorname{Ext}_{\mathrm{z}}(X, Y)$, where $\bar{f}$ is the image of $f$ in $\operatorname{coker}\left(\theta_{y}\right)$ and $(X, x)$ is any torsion free, rank 1 abelian group $X$ with nonzero element $x$ satisfying $\operatorname{char}(x)=$ $\left(a_{1}, \cdots a_{n}, \cdots\right)$.

Observe that in the particular case that $Y$ is free and $y$ is a generator, Proposition 1.1 asserts that

$$
\operatorname{Ext}_{\mathbf{Z}}(X, \mathbf{Z}) \rightarrow\left(\mathrm{IIZ} / p_{i}^{a_{i}}\right) / \Delta(\mathbf{Z})
$$

so that two extension functions determine isomorphic extensions if and only if they differ by a constant.

We recall that if $A$ is a finite rank, torsion free abelian group, then $\Lambda \otimes Z_{p}$ is a direct sum of $Z_{p}$ 's and $Q_{p}$ 's for every prime $p$, since $\operatorname{Ext}_{\mathbf{z}}^{1}\left(Q_{p}, Z_{p}\right)=0$ (where $Z_{p}$ denotes the $p$-adic integers and $Q_{p}$ denotes the field of $p$-adic numbers). We say that $\left\{x_{1}, \cdots, x_{n}\right\}$ in $A \otimes Z_{p}$ is a "basis" if $A \otimes \mathbf{Z}_{p}$ is the internal direct sum of the pure $\mathbf{Z}_{p}$ modules generated by the $x_{i}$ 's:

$$
A \otimes \mathbf{Z}_{p} \stackrel{\sim}{\rightarrow} \mathbf{Z}_{p} \cdot x_{i_{1}} \oplus \cdots \oplus \mathbf{Z}_{p} \cdot x_{i_{k}} \oplus Q_{p} \cdot x_{i_{k+1}} \oplus \cdots \oplus Q_{p} \cdot x_{i_{n}}
$$

A "matrix sequence" for a finite rank, torsion free abelian group $A$ is a sequence of matrices $\left\{M_{i}\right\}$ expressing a given basis for $A \otimes Q$ in terms of bases for $A \otimes Z_{p_{i}}$ thus, if $A$ is rank $2,\left\{M_{i}\left(\begin{array}{l}1 \\ 0\end{array}\right), M_{i}\left(\begin{array}{l}0 \\ 1\end{array}\right)\right\}$ is the given basis for $A \otimes Q$ expressed in terms of a basis for $A \otimes \mathbf{Z}_{p_{i}}$. Such a matrix sequence determines the isomorphism class of $A$.

In the following proposition, we determine matrix sequences in terms of extension functions.

Proposition 1.2. Let $X$ and $Y$ be rank 1 , torsion free abelian groups, let $x$ in $X$ and $y$ in $Y$ be nonzero elements, and let $\operatorname{char}(x)=$ 
$\left(a_{1}, \cdots, a_{n}, \cdots\right)$ and $\operatorname{char}(y)=\left(b_{1}, \cdots, b_{n}, \cdots\right)$. For a given extension function $f$ in $\Pi^{\prime} \mathbf{Z} / p_{i}^{a_{i}}$, let

$$
0 \rightarrow Y \rightarrow A_{f} \rightarrow X \rightarrow 0
$$

be the extension $\varphi(X, x ; Y, y)^{-1}(\bar{f})$ in $\operatorname{Ext}_{\mathrm{z}}^{1}(X, Y)$. Then $A_{f}$ has a matrix sequence

$$
\left\{\left(\begin{array}{cc}
p_{i}^{b_{i}} & -f_{i} \\
0 & p_{i}^{a_{i}}
\end{array}\right)\right\}
$$

where $0 \leqq f_{i}<p_{i}^{a_{i}}$ with $f(i)=f_{i}$ in $\mathbf{Z} / p_{i}^{a_{i}}$ if $a_{i}, b_{i}<\infty$, where $f_{i}=0$ and $p_{i}^{b_{i}}$ designates 1 if $b_{i}=\infty$, and where $f_{i}=f(i)$ in $\mathbf{Z}_{p_{i}}$ and $p_{i}^{a_{i}}$ designates 1 if $a_{i}=\infty$.

Proof. The extension $\varphi(X, x ; Y, y)^{-1}(\bar{f})$ is obtained by pull-back from $0 \rightarrow Y \rightarrow Q \rightarrow Q / Y \rightarrow 0$ via the composition $X \rightarrow X / Z \cdot x \rightarrow Q / Y$. For notational convenience, we view $A_{f}=Q \times{ }_{Q / Y} X$ as a subgroup of $Q \oplus X$, containing $(1,0)=y$ and $(0, x)=x$.

For each $i$ with $b_{i}=\infty,\left\{y, p_{i}^{-a_{i}} y\right\}$ in $A_{f}$ is a basis for $A_{f} \otimes \mathbf{Z}_{p i}$. For each $i$ with $b_{i}, a_{i}<\infty,\left\{p_{i}^{-b_{i}} y, f_{i} p_{i}^{-a_{i}-b_{i}} y+p_{i}^{-a_{i}} y\right\}$ in $A_{f}$ is a basis for $A_{f} \otimes \mathbf{Z}_{p_{i}}$. For each $i$ with $b_{i}<\infty$ and $a_{i}=\infty f_{i, k} p_{i}^{-k-b_{i}} y+p_{i}^{-k} y$ is in $A_{f}$ for all $k>0$ where $f_{i} \equiv f_{i, k}\left(\bmod p_{i}^{k}\right)$, so that $\left\{p_{i}^{-b_{l}} y, f_{i} p_{i}^{-b_{i}} y+x\right\}$ is a basis for $A_{f} \otimes \mathbf{Z}_{p_{i}}$. Then $\left(\begin{array}{cc}p_{i}^{b_{i}} & -f_{i} \\ 0 & p_{i}^{a_{i}}\end{array}\right)$ is the matrix expressing the basis $\{y, x\}$ of $A_{f} \otimes Q$ in terms of the above basis for $A_{f} \otimes \mathbf{Z}_{p_{i}}$.

We next determine in terms of generators a standard model for the rank 2 , torsion free abelian group $A_{f}$ defined by the extension function $f$.

Proposition 1.3. Let $X$ and $Y$ be rank 1, torsion free abelian groups, let $x$ in $X$ and $y$ in $Y$ be nonzero elements, and let $\operatorname{char}(x)=$ $\left(a_{1}, \cdots, a_{n}, \cdots\right)$ and $\operatorname{char}(y)=\left(b_{1}, \cdots, b_{n}, \cdots\right)$. For a given extension function $f$ in $\Pi^{\prime} \mathrm{Z} / p_{i}^{a_{i}}$, the map

$$
i(y, x): A_{f}=Q \underset{Q / Y}{\times} X \rightarrow Q \oplus Q
$$

sending y to $(1,0)$ and $x$ to $(0,1)$ is an isomorphism onto that subgroup $A(f)$ of $Q \oplus Q$ generated by $\left(p_{i}^{b_{i}}, 0\right),\left(f_{i} p_{i}^{-a_{i}-b_{i}}, p_{i}^{-a_{i}}\right)$ whenever $a_{i}, b_{i}<\infty$; $\left(p_{i}^{-k}, 0\right),\left(0, p_{i}^{-a_{i}}\right)$ for all $k>0$, whenever $a_{i}<\infty, b_{i}=\infty ;\left(p_{i}^{-k}, 0\right),\left(0, p_{i}^{-k}\right)$ for all $k>0$, whenever $a_{i}=\infty=b_{i} . \quad\left(p_{i}^{-b_{i}}, 0\right),\left(f_{i, k} p_{i}^{-k-b_{i}}, p_{i}^{-k}\right)$ where $f_{i, k}$ in $\mathbf{Z}$ satisfies $f_{i, k} \equiv f_{i}\left(\bmod p_{i}^{k}\right)$ for all $k>0$, whenever $a_{i}=\infty, b_{i}<\infty$.

Proof. Since $A_{f}$ is torsion free and $\{y, x\}$ is a basis for $A_{f} \otimes Q$, $i(y, x)$ is an isomorphism of $A_{f}$ onto its image $i\left(A_{f}\right)$. As checked in the 
proof of Proposition 1.2, $A(f) \subset i\left(A_{f}\right)$. Moreover, one readily checks that char $(1,0))$ in $A(f)$ equals $\left(b_{1}, \cdots, b_{n}, \cdots\right)$ by determining the $p$ divisibility of $(1,0)$ in $A(f) \otimes \mathbf{Z}_{p}$ for all primes $p$. Therefore, $A(f) \cap$ $(Q \otimes 0)=i(Y)$. Furthermore, the inclusion $A(f) \subset i\left(A_{f}\right)$ determines $A(f) / A(f) \cap(Q \oplus 0) \subset i(X)$ with $\overline{(0,1)}=i(y, x)(x)$. This is also an isomorphism, since char $(\overline{0,1}))$ in $A(f) / A(f) \cap(Q \oplus 0)$ equals $\operatorname{char}(x)$ in $X$. Thus, $A(f)$ equals $i\left(A_{f}\right)$.

By employing the matrix classification for rank 2, torsion free abelian groups, we give below necessary and sufficient conditions for two extension functions $f$ and $g$ to determine isomorphic total groups $A_{f}$ and $A_{g}$. Because of the very ineffective nature of the Korosh-MalcevDerry matrix classification, the significance of Proposition 1.4 is the explicit necessary condition it provides.

Proposition 1.4. Let $X$ and $Y$ be rank 1 , torsion free abelian grroups, let $x$ in $X$ and $y$ in $Y$ be nonzero elements, and let $\operatorname{char}(x)=$ $\left(a_{1}, \cdots, a_{n}, \cdots\right)$ and $\operatorname{char}(y)=\left(b_{1}, \cdots, b_{n}, \cdots\right)$. Two extension functions $f, g$ in $\Pi^{\prime} Z / p_{i}^{a_{i}}$ determine isomorphic total groups $A_{f}$ and $A_{g}$ if and only if there exists a matrix $\left(\begin{array}{ll}\alpha & \beta \\ \gamma & \delta\end{array}\right)$ in $\operatorname{GL}(Q \oplus Q)$ and matrices $\left(\begin{array}{ll}\alpha_{i} & \beta_{i} \\ \gamma_{i} & \delta_{i}\end{array}\right)$ in $\mathrm{GL}\left(Y \otimes \mathbf{Z}_{p_{t}} \oplus X \oplus \mathbf{Z}_{p_{1}}\right)$ satisfying

$(1.4 .1)_{i} \quad\left(\begin{array}{cc}p_{i}^{b_{i}} & -f_{i} \\ 0 & p_{i}^{a_{i}}\end{array}\right) \cdot\left(\begin{array}{cc}\alpha & \beta \\ \gamma & \delta\end{array}\right)=\left(\begin{array}{cc}\alpha_{i} & \beta_{i} \\ \gamma_{i} & \delta_{i}\end{array}\right) \cdot\left(\begin{array}{cc}p_{i}^{b_{i}} & -g_{i} \\ 0 & p_{i}^{a_{i}}\end{array}\right)$

for all $i$. In particular, if $A_{f}$ is isomorphic to $A_{g}$, then there exists $\alpha, \beta, \gamma, \delta$ in $Q$ satisfying $\alpha \delta-\beta \gamma \neq 0$ such that $f$ and $g$ satisfy the following congruence condition for every $i$ with $b_{i}<\infty$ :

$$
p_{i}^{b_{i}} \cdot \beta-f_{i} \cdot \delta \equiv g_{i}\left(f_{i} p_{i}^{-b_{i}} \gamma-\alpha\right) \quad\left(\bmod p_{i}^{a_{i}}\right)
$$

Proof. The conditions $(1.4 .1)_{i}$ express the relationship between matrix sequences for bases $\{x, y\}$ and $\left\{x^{\prime}, y^{\prime}\right\}$ of $A_{f} \otimes Q \simeq Q \oplus Q \simeq$ $A_{g} \otimes Q$ related by $\left(\begin{array}{ll}\alpha & \beta \\ \gamma & \delta\end{array}\right)$ in $\operatorname{GL}(Q \oplus Q)$ in terms of bases $\left\{x_{i}, y_{i}\right\}$ and $\left\{x_{i}^{\prime}, y_{i}^{\prime}\right\} \quad$ of $\quad Y \otimes \mathbf{Z}_{p_{i}} \oplus X \otimes \mathbf{Z}_{p_{i}} \quad$ related by $\left(\begin{array}{ll}\alpha_{i} & \beta_{i} \\ \gamma_{i} & \delta_{i}\end{array}\right) \quad$ in $\mathrm{GL}\left(Y \otimes \mathbf{Z}_{p_{i}} \oplus X \otimes \mathbf{Z}_{p_{i}}\right)$. The necessity of conditions (1.4.1) is clear; their sufficiency is well known (see [2]). If $b_{i}<\infty$ and $\left(\begin{array}{ll}\alpha_{i} & \beta_{i} \\ \gamma_{i} & \delta_{i}\end{array}\right)$ is in $\mathrm{GL}\left(Y \otimes \mathbf{Z}_{p_{i}} \oplus X \otimes \mathbf{Z}_{p_{i}}\right)$ then $\beta_{i}$ is in $\mathbf{Z}_{p_{i}}$. With this in mind, conditions $(1.4 .2)_{i}$ quickly follow from $(1.4 .1)_{i}$. 
Since the 0 function, $g=0$, determines $A_{g} \simeq Y \oplus X$, conditions $(1.4 .2)_{i}$ determine useful necessary conditions an extension function $f$ must satisfy for $A_{f}$ to be isomophic to $Y \oplus X$.

The following definition formulizes the relationship between extension functions $f$ and $g$ which determine isomorphic total groups $A_{f}$ and $A_{g}$.

DEFINITION 1.5. Let characteristics $\left(a_{1}, \cdots, a_{n}, \cdots\right)$ and $\left(b_{1}, \cdots, b_{n}, \cdots\right)$ be given and let $f, g$ be elements of $\Pi^{\prime} \mathbf{Z} / p_{i}^{a_{i}}$ where $\Pi^{\prime}$ is the product over all $i$ with $b_{i}<\infty$. Then $f$ and $g$ are said to be of the same type with respect to $\left(b_{1}, \cdots, b_{n}, \cdots\right)$ if there exists $\left(\begin{array}{ll}\alpha & \beta \\ \gamma & \delta\end{array}\right)$ in

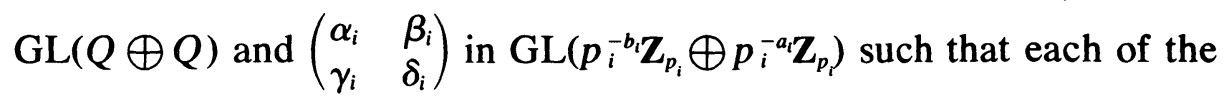
equations (1.4.1) holds, where $p_{i}^{-m} \mathbf{Z}_{p_{i}}$ is either $\mathbf{Z}_{p_{i}}$ or $Q_{p_{i}}$ depending on whether $m<\infty$ or $m=\infty$.

2. Applications. As a first step toward classifying those rank 2, torsion free abelian groups $A$ admitting a pure subgroup isomorphic to a given rak 1 group $Y$, we verify that the isomorphism class of $A / H$ is independent of the choice of pure subgroup $H$ isomorphic to $Y$. The proof of the following proposition was given to us by the referee.

Proposition 2.1. Let $Y$ be a rank 1, torsion free abelian group, and let $A$ be a rank 2, torsion free abelian group. For any two pure subgroups $H, H^{\prime}$ of $A$ isomorphic to $Y, A / H$ is isomorphic to $A / H^{\prime}$.

Proof. Let $x$ and $x^{\prime}$ be nonzero elements of $H$ and $H^{\prime}$ respectively. If there exists nonzero $m / n, m^{\prime} / n^{\prime}$ in $Q$ with $m / n \cdot x+$ $m^{\prime} / n^{\prime} \cdot x^{\prime}=0$ in $A$, then $H$ and $H^{\prime}$ are equal; namely, each is the pure subgroup generated by $n^{\prime} m x=-n m^{\prime} x^{\prime}$. Consequently, we may assume $\left\{x, x^{\prime}\right\}$ span $A \otimes Q$.

Define $H_{0}=\left\{r\right.$ in $Q \mid r x+s x^{\prime}$ is in $A$ for some $s$ in $\left.Q\right\}$. Then the map $A \rightarrow H_{0}$ sending $r x+s x^{\prime}$ to $r$ is surjective with kernel $H^{\prime}$. Similarly, if $H_{0}^{\prime}=\left\{s\right.$ in $Q \mid r x+s x^{\prime}$ is in $A$ for some $r$ in $\left.Q\right\}$, then $A \rightarrow H_{0}^{\prime}$ has kernel $H$. Therefore, it suffices to prove that $H_{0}$ is isomorphic to $H_{0}^{\prime}$.

Consider the inclusions $H \subset H_{0}$ sending $r x$ to $r$, and $H^{\prime} \subset H_{0}^{\prime}$ sending $s x^{\prime}$ to $s$. Then $H_{0} \rightarrow H_{0}^{\prime} / H^{\prime}$ sending $r$ to the class of some $s$ such that $r x+s x^{\prime}$ is in $A$ is well defined: if $r x+s^{\prime} x^{\prime}$ is also in $A$, then $\left(s-s^{\prime}\right) x^{\prime}$ is in $H^{\prime}$. Clearly, this map induces an isomorphism $H_{0} / H \rightarrow H_{0}^{\prime} / H^{\prime}$. Since $H_{0}$ and $H_{0}^{\prime}$ are rank 1 , torsion free with isomorphic subgroups $H$ and $H^{\prime}$ respectively and isomorphic quotient groups $H_{0} / H$ and $H_{0}^{\prime} / H^{\prime}, H_{0}$ is isomorphic to $H_{0}^{\prime}$. 
The following theorem summarizes the discussion of $\S 1$ together with Proposition 2.1.

THEOREM 2.2. Let $Y$ be a rank 1, torsion free abelian group and let $y$ in $Y$ be a nonzero element. Then there is a natural 1-1 correspondence with explicit inverse

$$
\Phi(Y, y): E(Y) \rightarrow T(Y, y)
$$

between the set $E(Y)$ of isomorphism classes of rank 2, torsion free abelian groups with a pure subgroup isomorphic to $Y$ and the set $T(Y, y)$ of pairs consisting of

(i) the type of some characteristic $\left(a_{1}, \cdots, a_{n}, \cdots\right)$

(ii) the type with respect to char( $y)$ of some extension function $f$ in $\Pi^{\prime} \mathbf{Z} / p_{i}^{a_{i}}$, which transforms to $\pm p_{1}^{c_{1}} \cdots p_{n}^{c_{n}} \cdots f$ in $\Pi^{\prime} \mathbf{Z} / p_{i}^{a_{i}+c_{i}}$ if all $c_{i} \geqq 0$ and $\sum c_{t}<\infty$.

Proof. We define $\Phi(Y, y)(A)$ for $A$ in $E(Y)$ as follows. Choose a rank 1, pure subgroup $H$ of $A$ with $O \neq h$ in $H$ such that $\operatorname{char}(h)=$ $\operatorname{char}(y)=\left(b_{1}, \cdots, b_{n}, \cdots\right)$. Let $x$ in $A / H$ be any nonzero element with $\operatorname{char}(x)=\left(a_{1}, \cdots, a_{n}, \cdots\right)$. Let $f$ in $\Pi^{\prime} \mathbf{Z} / p_{i}^{a_{i}}$ be an extension function representing $\varphi(A / H, x ; H, h)(0 \rightarrow H \rightarrow A \rightarrow A / H \rightarrow 0)$ as in Proposition 1.1. Define $\Phi(Y, y)(A)=\{\operatorname{char}(x), f\}$.

By Proposition 2.1, the type of $\operatorname{char}(x)$ is well defined, independent of the choice of $H$ or $x$. By Proposition 1.4, type of $f$ depends at most upon choices for $A / H, x, H, h$, since $A$ is isomorphic to $A_{f}$. If $H^{\prime}$ is another rank 1, pure subgroup of $A$ with $O \neq h^{\prime}$ in $H^{\prime}$ such that $\operatorname{char}\left(h^{\prime}\right)=\operatorname{char}(y)$, if $x^{\prime}$ in $A / H^{\prime}$ is chosen with $\operatorname{char}\left(x^{\prime}\right)=\operatorname{char}(x)$, and if $f^{\prime}$ in $\Pi^{\prime} Z / p_{i}^{a_{i}}$ represents

$$
\varphi\left(A / H^{\prime}, x^{\prime} ; H^{\prime}, h^{\prime}\right)\left(0 \rightarrow H^{\prime} \rightarrow A \rightarrow A / H^{\prime} \rightarrow 0\right),
$$

then

$$
\left\{\left(\begin{array}{cc}
p_{i}^{b_{i}} & -f_{i} \\
0 & p_{i}^{a_{i}}
\end{array}\right)\right\}, \quad\left\{\left(\begin{array}{cc}
p_{i}^{b_{i}} & -f_{i}^{\prime} \\
0 & p_{i}^{a_{i}}
\end{array}\right)\right\}
$$

are each matrix sequences for $A$ by Proposition 1.2. Hence, by Proposition $1.4, f$ and $f^{\prime}$ have the same type. Finally, for a given rank 1, pure subgroup $H$ of $A$ with $O \neq h$ in $H$ such that $\operatorname{char}(h)=\operatorname{char}(y)$, let $x^{\prime}=m x$ be an integer nonzero multiple of $x$ in $A / H$. As an extension function for $0 \rightarrow H \rightarrow A \rightarrow A / H \rightarrow 0, f$ may be viewed as a classifying map $f:(A / H) / Z \cdot x \rightarrow Q / H$; this induces the classifying map $f^{\prime}:(A / H) / \mathbf{Z} \cdot x^{\prime} \rightarrow Q / H$. Under the identifications 
$\operatorname{Hom}((A / H) / \mathbf{Z} \cdot x, Q / H)=\Pi^{\prime} \mathbf{Z} / p_{i}^{a_{i}}$

$\operatorname{Hom}\left((A / H) / \mathbf{Z} \cdot x^{\prime}, Q / H\right)=\Pi^{\prime} \mathbf{Z} / p_{i}^{a_{i}+c_{i}}$ with $c_{i}=\max \left\{c \mid p_{i}^{c}\right.$ divides $\left.m\right\}$, $f^{\prime}= \pm p_{1}^{c_{1}} \cdots p_{n}^{c_{n}} \cdots f$ in $\Pi^{\prime} \mathbf{Z} / p_{i}^{a_{i} c_{i}}$.

The naturality of $\Phi(Y, y)$ with respect to $Y$ and $y$ follows from the naturality of extension functions as given in Proposition 1.1. The fact that $\Phi(Y, y)$ is injective follows from the fact that $A$ is isomorphic to $A_{f}$ if $\Phi(Y, y)(A)=\left\{\left(a_{1}, \cdots a_{n}, \cdots\right), f\right\}$. The fact that $\Phi(Y, y)$ is surjective follows from Proposition 1.2, since $\Phi(Y, y)\left(A_{f}\right)=\left\{\left(a_{1}, \cdots, a_{n}, \cdots\right), f\right\}$. Finally, the explicit inverse of $\Phi(Y, y)$ is given in Proposition 1.3.

We say that a torsion free abelian group $A$ is homogeneous of type $\left(b_{1}, \cdots, b_{n}, \cdots\right)$ if for every nonzero $x$ in $A$, the type of char $(x)$ equals the type of $\left(b_{1}, \cdots, b_{n}, \cdots\right)$. Using Theorem 2.2, we "list" all rank 2, torsion free abelian groups which are homogeneous of type

$$
\left(b_{1}, \cdots, b_{n}, \cdots\right) \text {. }
$$

THEOREM 2.3. Let $\left(b_{1}, \cdots, b_{n}, \cdots\right)$ be a characteristic and let $Y$ be a rank 1, torsion free abelian group with $O \neq y$ in $Y$ such that $\operatorname{char}(y)=\left(b_{1}, \cdots, b_{n}, \cdots\right)$. Then the set of all rank 2 , torsion free abelian groups homogeneous of type $\left(b_{1}, \cdots, b_{n}, \cdots\right)$ is the subset of $E(Y)$ consisting of those $A$ in $E(Y)$ such that $\Phi(Y, y)(A)=$ $\left\{\left(a_{1}, \cdots, a_{n}, \cdots\right), f\right.$ in $\left.\Pi^{\prime} \mathbf{Z} / p_{i}^{a^{\prime}}\right\}$ satisfies

(i) if $b_{i}=\infty$, then $a_{i}=\infty$;

(ii) if $b_{i}<\infty$ and $a_{i}=\infty$, then $f_{i}$ in $\mathbf{Z}_{p_{i}}$ is not rational;

(iii) for all but finitely many $i$ with $b_{i}<\infty, a_{i} \geqq b_{i}$ and $p_{i}^{b_{i}} \mid f_{i}$;

(iv) for every nonzero pair of integers $\{m, n\}$, there are only finitely many $i$ with $a_{i}>b_{i}, p_{i}^{b_{i}} \mid f_{i}$, and $m-n f_{i} / p_{i}^{b_{i}}$ divisible by $p_{i}$.

Proof. The set of isomorphism classes of rank 2, torsion free abelian groups homogeneous of type $\left(b_{1}, \cdots, b_{n}, \cdots\right)$ is clearly a subset of $E(Y)$. To prove the theorem, it suffices to prove the following for a given pair $\left\{\left(a_{1}, \cdots, a_{n}, \cdots\right), f\right.$ in $\left.\Pi^{\prime} \mathbf{Z} / p_{i}^{a_{i}}\right\}$ : the characteristic of every nonzero element $(m, n)$ in $A(f) \cap \mathbf{Z} \oplus \mathbf{Z}$ as an element of $A(f)$ has the same type as $\left(b_{1}, \cdots, b_{n}, \cdots\right)$ iff conditions (i) -(iv) are satisfied (where $A(f)$ is given in Proposition 1.3.). We write

$$
(m, n)=\left(m p_{i}^{b_{i}}-n f_{i}\right)\left(p_{i}^{-b_{i}}, 0\right)+n p_{i}^{a_{i}}\left(f_{i} p_{i}^{-a_{l}-b_{l}}, p_{i}^{-a_{i}}\right)
$$

in $A(f) \otimes \mathbf{Z}_{p i}$, where $p_{i}^{\infty}=1$ and $\dot{f}_{i}=0$ if $b_{i}=\infty$. We observe for any $k>0$ that $(m, n)$ is divisible by $p_{i}^{k}$ in $A(f)$ iff $(m, n)$ is divisible by $p_{i}^{k}$ in $A(f) \otimes \mathbf{Z}_{p_{i}}$.

If $b_{i}=\infty$, then every $(m, n)$ in $A(f) \cap \mathbf{Z} \oplus \mathbf{Z}$ is infinitely $p_{i}$ divisible (as an element of $A(f))$ iff $a_{i}=\infty$. If $b_{i}<\infty$, then $(m, n)$ in 
$A(f) \cap \mathbf{Z} \oplus \mathbf{Z}$ is not infinitely $p_{i}$ divisible iff either $a_{i}<\infty$ or $a_{i}=\infty$ and $m p_{i}^{b_{i}}-n f_{i} \neq 0$. Consequently, (i) and (ii) are equivalent to the condition that one nonzero element of $A(f)$ is infinitely $p_{i}$ divisible iff all nonzero elements of $A(f)$ are.

$(m, n)$ in $A(f) \cap \mathbf{Z} \oplus \mathbf{Z}$ is divisible by $p_{i}^{b_{i}}$ for all but finitely many $i$ with $b_{i}<\infty$ iff $n=0$ or for all but finitely many $i$ with $b_{i}<\infty, a_{i} \geqq b_{i}$ and $p_{i}^{b_{1}} \mid f_{1} . \quad(m, n)$ is not divisible by $p_{i}^{b_{i}+1}$ for all but finitely many $i$ with $b_{1}<\infty$ iff for all but finitely many $i$ with $a_{i}>b_{i}, m p_{i}^{b i}-n f_{i}$ is not divisible by $p_{i}^{b_{i}+1}$. Consequently, (iii) and (iv) are equivalent to the condition that the characteristic of any nonzero element of $A(f)$ differs from $\left(b_{1}, \cdots, b_{n}, \cdots\right)$ at only finitely many $i$ with $b_{i}$.

To show how explicit Theorem 2.3 is, we provide the following simple example.

EXAMPLE 2.4. Let $\left(b_{1}, \cdots, b_{n}, \cdots\right)$ be a characteristic such that $b_{i}<\infty$ for infinitely many $i$. Let $Y$ be a rank 1 , torsion free abelian group with nonzero element $y$ with $\operatorname{char}(y)=\left(b_{1}, \cdots, b_{n}, \cdots\right)$. Then for any positive integer $k$,

$$
\left.\Phi(Y, y)^{-1}\left(\left\{b_{1}+k, \cdots, b_{n}+k, \cdots\right), f=i \cdot p_{i}^{b_{i}} \text { in } \Pi^{\prime} \mathbf{Z} / p_{i}^{b_{i}+k}\right\}\right)
$$

is an indecomposable, rank 2 , torsion free abelian group homogeneous of type $\left(b_{1}, \cdots, b_{n}, \cdots\right)$.

Proof. If $A$ in $E(Y)$ is homogeneous and decomposable, then $A \simeq Y \oplus Y$ so that the quotient type of $A$ (i.e., the first invariant of Theorem 2.2) must be the type of $\left(b_{1}, \cdots, b_{n}, \cdots\right)$. Consequently, to check the example it suffices to verify conditions (i) -(iv) of Theorem 2.3 for $\left\{\left(b_{1}+k, \cdots, b_{n}+k, \cdots\right), f=i \cdot p_{i}^{b_{i}}\right\}$. Conditions (i), (ii), and (iii) are immediate. To verify condition (iv), we observe that for sufficiently large $i \quad 0<n \cdot i-m<p_{i}$ by the prime number theorem.

In particular, Example 2.4 gives examples of nonfree homogeneous groups $A$, with type $A=(0, \cdots, 0 \cdots)$, such that $A \otimes \mathbf{Z}_{p}$ is a free $\mathbf{Z}_{p}$ module for all primes $p$. These groups are called locally free.

As another application of Theorem 2.2, we determine the subset $E(\mathbf{Z}) \cap E(Y)$ of $E(Y)$.

Proposition 2.5. Let $\left(b_{1}, \cdots, b_{n}, \cdots\right)$ be a characteristic and let $Y$ be a rank 1 , torsion free abelian group with $0 \neq y$ in $Y$ such that $\operatorname{char}(y)=\left(b_{1}, \cdots, b_{n}, \cdots\right)$. Then the subset $E(\mathbf{Z}) \cap E(Y)$ of $E(Y)$ con sists of those $A$ in $E(Y)$ such that $\Phi(Y, y)(A)=\left\{\left(a_{1}, \cdots, a_{n}, \cdots\right), f\right.$ in $\left.\Pi^{\prime} \mathbf{Z} / p_{i}^{a_{i}}\right\}$ satisfies :

(i) if $b_{i}=\infty$, then $a_{i}<\infty$;

(ii) for all but finitely many $i$ with $a_{i}, b_{i}>0, p_{i}$ does not divide $f_{i}$; 
(iii) there exists a nonzero pair of integers $\{m, n\}$ such that $m p_{i}^{b_{i}}-n f_{i} \neq 0$ whenever $a_{i}=\infty$, and $m-n f_{i}$ is divisible by $p_{i}$ for only finitely many $i$ with $b_{i}=0$ and $a_{i}>0$.

Proof. For $Y$ cyclic, conditions (i), (ii), and (iii) are immediately satisfied for any pair of invariants $\left\{\left(a_{1}, \cdots, a_{n}, \cdots\right), f\right\}$. We may thus assume $Y$ is not cyclic. As in the proof of Theorem 2.3, to prove the proposition it suffices to prove that $(m, n)$ in $A(f) \cap \mathbf{Z} \oplus \mathbf{Z}$ generates a pure cyclic subgroup of $A(f)$ iff (i) and (ii) are satisfied and $\{m, n\}$ satisfies (iii). Since $Y$ is assumed not to be cyclic, we need only consider $(m, n)$ with $n \neq 0$. We write

$$
(m, n)=\left(m p_{i}^{b_{i}}-n f_{i}\right)\left(p_{i}^{-b_{i}}, 0\right)+n p_{i}^{a_{i}}\left(f_{i} p_{i}^{-a_{i}-b_{i}}, p_{i}^{-a_{i}}\right) .
$$

Now, $(m, n)$ with $n \neq 0$ is not infinitely divisible in $A(f) \otimes \mathbf{Z}_{p_{1}}$ by $p_{i}$ iff whenever $b_{i}=\infty$, then $a_{i}<\infty$; and whenever $a_{i}=\infty$, then $m p_{i}^{b_{i}}-n f_{i} \neq 0$. Furthermore, $(m, n)$ with $n \neq 0$ is divisible by only finitely many $p_{i}$ iff for only finitely many $i$ with $b_{i}, a_{i}>0 f_{i}$ is divisible by $p_{i}$; and for only finitely many $i$ with $b_{i}=0, a_{i}>0 m-n f_{i}$ is divisible by $p_{i}$.

\section{REFERENCES}

1. R. A. Beaumont and R. S. Pierce, Torsion free groups of rank two, Mem. Amer. Math. Soc. Nr. 38.

2. L. Fuchs, Infinite abelian groups, Vol. II, Academic Press, New York and London, 1973.

Received January 23, 1974. Partially supported by the U.S. - France Exchange of Scientists Program.

PRINCETON UNIVERSITY 


\section{PACIFIC JOURNAL OF MATHEMATICS}

\section{EDITORS}

RICHARD ARENS (Managing Editor)

University of California

Los Angeles, California 90024

\section{J. DugundJI}

Department of Mathematics University of Southern California Los Angeles, California 90007

D. Gilbarg and J. Milgram

Stanford University

Stanford, California 94305

\section{ASSOCIATE EDITORS}
E. F. BECKENBACH
B. H. NeumanN
F. WoLF
K. YoshiDA

\section{SUPPORTING INSTITUTIONS}

\author{
UNIVERSITY OF BRITISH COLUMBIA \\ CALIFORNIA INSTITUTE OF TECHNOLOGY \\ UNIVERSITY OF CALIFORNIA \\ MONTANA STATE UNIVERSITY \\ UNIVERSITY OF NEVADA \\ NEW MEXICO STATE UNIVERSITY \\ OREGON STATE UNIVERSITY \\ UNIVERSITY OF OREGON \\ OSAKA UNIVERSITY
}

\author{
UNIVERSITY OF SOUTHERN CALIFORNIA \\ STANFORD UNIVERSITY \\ UNIVERSITY OF TOKYO \\ UNIVERSITY OF UTAH \\ WASHINGTON STATE UNIVERSITY \\ UNIVERSITY OF WASHINGTON \\ AMERICAN MATHEMATICAL SOCIETY
}

The Supporting Institutions listed above contribute to the cost of publication of this Journal, but they are not owners or publishers and have no responsibility for its contents or policies.

Mathematical papers intended for publication in the Pacific Journal of Mathematics should be in typed form or offset-reproduced (not dittoed), double spaced with large margins. Underline Greek letters in red, German in green, and script in blue. The first paragraph or two must be capable of being used separately as a synopsis of the entire paper. Items of the bibliography should not be cited there unless absolutely necessary, in which case they must be identified by author and Journal, rather than by item number. Manuscripts, in duplicate, may be sent to any one of the four editors. Please classify according to the scheme of Math. Reviews, Index to Vol. 39. All other communications should be addressed to the managing editor, or Elaine Barth, University of California, Los Angeles, California, 90024.

100 reprints are provided free for each article, only if page charges have been substantially paid. Additional copies may be obtained at cost in multiples of 50 .

The Pacific Journal of Mathematics is issued monthly as of January 1966. Regular subscription rate: $\$ 72.00$ a year (6 Vols., 12 issues). Special rate: $\$ 36.00$ a year to individual members of supporting institutions.

Subscriptions, orders for back numbers, and changes of address should be sent to Pacific Journal of Mathematics, 103 Highland Boulevard, Berkeley, California, 94708.

PUBLISHED BY PACIFIC JOURNAL OF MATHEMATICS, A NON-PROFIT CORPORATION Printed at Jerusalem Academic Press, POB 2390, Jerusalem, Israel.

$$
\begin{gathered}
\text { Copyright (C) } 1975 \text { Pacific Journal of Mathematics } \\
\text { All Rights Reserved }
\end{gathered}
$$




\section{Pacific Journal of Mathematics}

\section{Vol. 58, No. $2 \quad$ April, 1975}

Zvi Artstein and John Allen Burns, Integration of compact set-valued functions . . . . . . . . . 297

Mark Benard, Characters and Schur indices of the unitary reflection group $[321]^{3} \ldots \ldots \ldots . .309$

Simeon M. Berman, A new characterization of characteristic functions of absolutely continuous

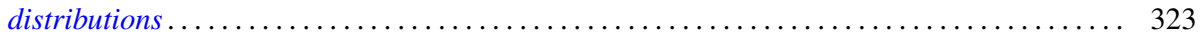

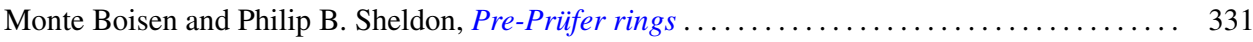

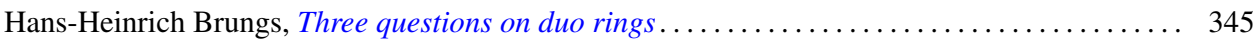

Iracema M. Bund, Birnbaum-Orlicz spaces of functions on groups................. 351

John D. Elwin and Donald R. Short, Branched immersions between 2-manifolds of higher

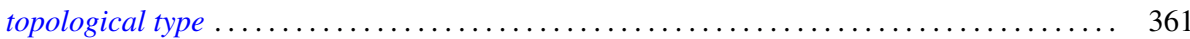

Eric Friedlander, Extension functions for rank 2, torsion free abelian groups . .......... 371

Jon Froemke and Robert Willis Quackenbush, The spectrum of an equational class of

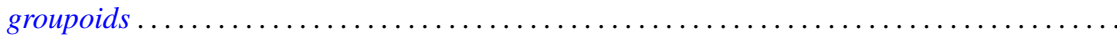

Barry J. Gardner, Radicals of supplementary semilattice sums of associative rings ...........

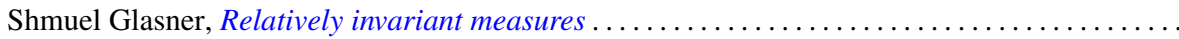

George Rudolph Gordh, Jr. and Sibe Mardesic, Characterizing local connectedness in inverse

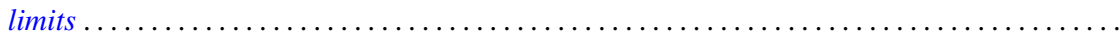

Siegfried Graf, On the existence of strong liftings in second countable topological spaces......

Stanley P. Gudder and D. Strawther, Orthogonally additive and orthogonally increasing

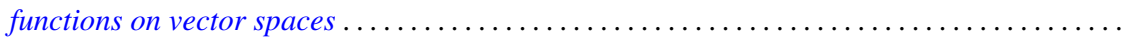

Darald Joe Hartfiel and Carlton James Maxson, A characterization of the maximal monoids and

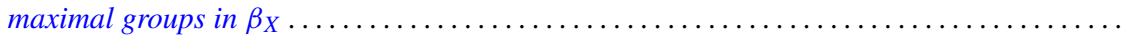

Robert E. Hartwig and S. Brent Morris, The universal flip matrix and the generalized faro-shuffle. .

William Emery Haver, Mappings between ANRs that are fine homotopy equivalences. .

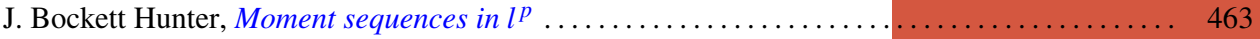

Barbara Jeffcott and William Thomas Spears, Semimodularity in the completion of a poset.... 467

Jerry Alan Johnson, A note on Banach spaces of Lipschitz functions . . . . . . . . . . . . 475

David W. Jonah and Bertram Manuel Schreiber, Transitive affine transformations on

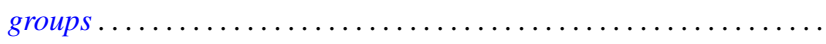

Karsten Juul, Some three-point subset properties connected with Menger's characterization of

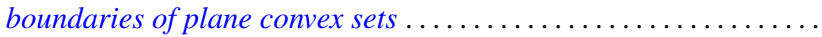

Ronald Brian Kirk, The Haar integral via non-standard analysis . . . . . . . . . . . . . 517

Justin Thomas Lloyd and William Smiley, On the group of permutations with countable

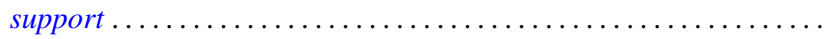

Erwin Lutwak, Dual mixed volumes .................................. 531

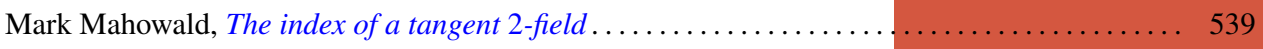

Keith Miller, Logarithmic convexity results for holomorphic semigroups . . . . . . . . . . . . 549

Paul Milnes, Extension of continuous functions on topological semigroups . . . . . . . . . . 553

Kenneth Clayton Pietz, Cauchy transforms and characteristic functions ................ 563

James Ted Rogers Jr., Whitney continua in the hyperspace $C(X) \ldots \ldots \ldots \ldots \ldots \ldots \ldots \ldots .569$

Jean-Marie G. Rolin, The inverse of a continuous additive functional . . . . . . . . . . . . 585

William Henry Ruckle, Absolutely divergent series and isomorphism of subspaces . ........ 605

Rolf Schneider, A measure of convexity for compact sets . ..................... 617

Alan Henry Schoenfeld, Continous measure-preserving maps onto Peano spaces .......... 627

V. Merriline Smith, Strongly superficial elements .......................... 643

Roger P. Ware, A note on quadratic forms over Pythagorean fields . . . . . . . . . . . . . . 651

Roger Allen Wiegand and Sylvia Wiegand, Finitely generated modules over Bezout rings . . . . 655

Martin Ziegler, A counterexample in the theory of definable automorphisms . . . . . . . . . 665 